\title{
Best Response Dynamics in Finite Games with Additive Aggregation*
}

\author{
Nikolai S. Kukushkin \\ Russian Academy of Sciences, Dorodnicyn Computing Center \\ 40, Vavilova, Moscow 119333 Russian Federation \\ E-mail: ququns@pochta.ru
}

\begin{abstract}
If in a finite strategic game all strategies are scalar, each player is only affected by the sum of the partners" choices, and one of three "single crossing" conditions is satisfied, then every best response improvement path leads to a Nash equilibrium. Journal of Economic Literature Classification Number: C 72.
\end{abstract}

*Financial support from the Russian Foundation for Basic Research (grants 99-01-01238 and 0201-00854), from the Russian state program for the support of the leading scientific schools (grant 0015-96118), and from Generalitat Valenciana (grant INV 00-08-16) is acknowledged. I thank Francisco Marhuenda and Carmen Herrero for procuring the latter grant, and Universidad de Alicante, Departamento de Fundamentos del Análisis Económico, for its hospitality. Helpful comments by an anonymous referee and an associate editor are also appreciated. 


\section{Introduction}

Individual myopic adaptation in strategic games has been studied since the time of A. Cournot. An important step was made recently by Monderer and Shapley (1996a), who established a link between unilateral improvement dynamics and "potential functions" in finite games and produced an impressive list of games having the finite improvement path (FIP) property, i.e., where every unilateral improvement path eventually leads to a Nash equilibrium. Milchtaich (1996) introduced a weaker finite best response improvement path (FBRP) property and showed that it is present in some games without FIP. Kukushkin (1999) suggested the use of the language of binary relations: both FIP and FBRP are easily restated as the acyclicity of the appropriate individual improvement relations. A potential is also understood as a strict order rather than a numeric function.

This paper applies the Monderer-Shapley-Milchtaich approach to a class of strategic games with natural algebraic properties. First, we assume that all strategies are scalar and each player is only affected by the sum of the partners' choices. This class of games was recognized and axiomatically characterized by Selten (1970, Chapters 8 and 9), and includes economic models such as the private provision of a public good (bad) and the Cournot oligopoly.

Second, we only consider finite games. Since no market in the world trades in infinitesimal (or indefinitely large, for that matter) quantities of any goods, this feature cannot be called unrealistic. The usual argument is that a continuous model is a convenient approximation to a discrete one when admissible points are dense enough on the real line. This paper shows that sometimes a discrete model may be more convenient. A similar treatment of continuous games is actually possible (Kukushkin, 2000a), but much more difficult technically.

Finally, a monotonicity condition is imposed in each theorem. Two conditions are quite general in their nature and were recognized as such by Bulow et al. (1985), who called them strategic complements and strategic substitutes, respectively. Both conditions are met in economic models quite often, see, e.g., Fudenberg and Tirole (1991, Section 12.3) and references there. The third only makes sense in games with additive aggregation; it was first formulated in the Cournot oligopoly model $\left(P^{\prime}-C^{\prime \prime}<0\right)$ and is often considered indispensable for the stability of equilibria. It seems appropriate, therefore, to stress that here the condition is only imposed in 
Theorem 3, where it is coupled with symmetry as in the seminal papers by McManus (1962, 1964).

Individual adaptation has been studied in games with strategic complementarities. Topkis (1979, Algorithm I) proved the convergence of best response improvement paths starting from the very bottom (or the very top) of the strategy profiles space. Vives (1990) extended the result to paths starting "very high" or "very low". Milgrom and Roberts (1990) considered rather general adaptive learning processes, but did not study their convergence. Friedman and Mezzetti (2001) proved that if the strategy sets are finite chains, then every strategy profile is connected to a Nash equilibrium with a unilateral improvement path; they called the property "weak FIP." Here we are interested in convergence to an equilibrium of all best response improvement paths, and we have to additionally assume additivity.

As to improvement dynamics in games with strategic substitutes, a very recent paper by Dubey et al. (2002), which treats strategic complements and substitutes uniformly, seems the only relevant reference. A comparison with this paper can be found in Section 7.9.

From a purely technical viewpoint, there are two principal ways to prove acyclicity. Sometimes, one can immediately derive a contradiction from the assumed existence of an improvement cycle, as in Theorem 1 below; alternatively, an explicit definition of a potential, i.e., of a strict order on the set of strategy profiles such that every best response improvement pushes the current profile upwards, can be obtained, as in Theorem 2. In Theorem 3, an intermediate course is taken: an explicit "quasipotential" is defined, i.e., a preorder such that every best response improvement either pushes the profile upwards or leaves it on the same level, and, when every player has made an improvement, the profile inevitably goes upwards.

The next section contains basic definitions and notation. In Section 3, the three main theorems are formulated and one of them proved. More complicated proofs of Theorems 2 and 3 are deferred to Sections 4 and 5, respectively. Section 6, inserted on a suggestion of the associate editor, describes relationships between the notions of an order potential, used in this paper, and of a numeric potential, as introduced by Monderer and Shapley (1996a). A discussion of possible extensions and open problems in Section 7 concludes the paper. 


\section{Basic Notions}

A strategic game $\Gamma$ is defined by a finite set of players $N$, and strategy sets $X_{i}$ and utility functions $u_{i}$ on $X=\prod_{i \in N} X_{i}$ for all $i \in N$. Throughout the paper only finite sets $X_{i}$ are considered. $\Gamma$ is called a game with additive aggregation (an AA game) if each $X_{i}$ is a subset of the real line $\left(X_{i} \subseteq \mathbb{R}\right)$ and, for each $i \in N$, there exists a function $V_{i}\left(s_{i}, x_{i}\right)$ such that $u_{i}(x)=V_{i}\left(\sum_{j \neq i} x_{j}, x_{i}\right)$. An AA game is regular if every $X_{i}$ is the set of integers satisfying $a_{i} \leq x_{i} \leq b_{i}$.

Remark. From the viewpoint of possible interpretations, perhaps, only regular games deserve attention; however, we do not assume regularity where it is not necessary.

For every AA game, player $i \in N$, and $x \in X$, we denote $\tau(x)=\sum_{i \in N} x_{i}$ and $\sigma_{i}(x)=\sum_{j \neq i} x_{j}\left[\right.$ note that $\tau(x)=\sigma_{i}(x)+x_{i}$ for each $\left.i \in N\right]$; we also denote $T=\tau(X)$ and $S_{i}=\sigma_{i}(X)$, and define the best response correspondence:

$$
R_{i}\left(s_{i}\right)=\underset{x_{i} \in X_{i}}{\operatorname{Argmax}} V_{i}\left(s_{i}, x_{i}\right)
$$

[in a finite game, $R_{i}\left(s_{i}\right) \neq \emptyset$ for each $\left.s_{i} \in S_{i}\right]$.

We introduce a few binary relations on $X(y, x \in X, i \in N)$ :

$$
\begin{gathered}
y \triangleright_{i} x \Longleftrightarrow\left[y_{-i}=x_{-i} \& u_{i}(y)>u_{i}(x) \& y_{i} \in R_{i}\left(\sigma_{i}(x)\right)\right] ; \\
y \triangleright x \Longleftrightarrow \exists i \in N\left[y \triangleright_{i} x\right] .
\end{gathered}
$$

A Cournot path is a (finite or infinite) sequence $\left\{x^{k}\right\}_{k=0,1, \ldots}$ such that $x^{k+1} \triangleright x^{k}$ whenever $x^{k+1}$ is defined. A Cournot cycle is a Cournot path such that $x^{m}=x^{0}$ for some $m>0$.

For a finite game, the absence of Cournot cycles obviously means that every Cournot path, if continued whenever possible, ends at a Nash equilibrium. Thus, the existence of an equilibrium is implied.

For all admissible $s_{i}, x_{i}$, and $\delta>0$, we define

$$
D_{i}\left(s_{i}, x_{i}, \delta\right)=V_{i}\left(s_{i}, x_{i}+\delta\right)-V_{i}\left(s_{i}, x_{i}\right)
$$

The increment helps to formulate various useful properties. If $D_{i}\left(s_{i}, x_{i}, \delta\right)$ decreases in $x_{i}$, the utility function is concave in own variable; however, this property is useless in discrete models studied in this paper. If the increment increases in $s_{i}$, there is Topkis's 
increasing differences property, which implies that the best response correspondence $R_{i}$ is increasing in the sense of Veinott (Topkis, 1978); if every $V_{i}$ has the property, a Nash equilibrium exists by the Tarski (1955) fixed point theorem. If the increment decreases in $s_{i}$ for each $i \in N$, the existence of a Nash equilibrium follows from the main theorem of Kukushkin (1994); the proof of the latter was based on a construction invented by Novshek (1985) for continuous games.

Finally, in a regular AA game, the situation deserves attention when $D_{i}\left(s_{i}, x_{i}, \delta\right)$ increases in a diagonal direction, with $x_{i}$ decreasing, $s_{i}$ increasing, and their sum keeping constant. Kukushkin (2000b) established the existence of an equilibrium in quasisymmetric discrete games with this property of utilities, extending the results obtained by McManus $(1962,1964)$ for symmetric continuous Cournot models. It was also noted in that paper that the condition can be regarded as innocuous in the context of the voluntary provision of a public good: it is satisfied if no increase in the consumption of the private good could increase its relative value to the consumer.

The three main theorems of this paper establish the absence of Cournot cycles under some ordinal versions of the three above properties, each of which could be named "single crossing" (Milgrom and Shannon, 1994).

\section{Theorems}

An AA game satisfies the strategic complements condition if

$$
D_{i}\left(s_{i}, x_{i}, \delta\right) \geq 0 \Rightarrow D_{i}\left(s_{i}+\Delta, x_{i}, \delta\right) \geq 0
$$

for all $i \in N$ and all admissible $s_{i}, x_{i}$, and $\delta, \Delta \geq 0$.

Theorem 1. No AA game with strategic complements admits a Cournot cycle.

Proof. Let $x^{0}, x^{1}, \ldots, x^{m}=x^{0}$ be a Cournot cycle. We pick $k$ such that $\tau\left(x^{k}\right)$ maximizes $\tau(x)$ on the cycle. Let $x^{k+1} \triangleright_{i} x^{k}$; then $x_{i}^{k+1} \in R_{i}\left(\sigma_{i}\left(x^{k}\right)\right), x_{i}^{k} \notin R_{i}\left(\sigma_{i}\left(x^{k}\right)\right)$, and $x_{i}^{k+1}<x_{i}^{k}$. On the other hand, $x_{i}^{k}$ must equal $x_{i}^{h+1} \in R_{i}\left(\sigma_{i}\left(x^{h}\right)\right)$ for some $h$; then $D_{i}\left(\sigma_{i}\left(x^{h}\right), x_{i}^{k+1}, \delta\right) \geq 0$ for $\delta=x_{i}^{k}-x_{i}^{k+1}>0$. Now $\sigma_{i}\left(x^{h}\right)=\sigma_{i}\left(x^{h+1}\right) \leq \sigma_{i}\left(x^{k}\right)$ by the choice of $k$, hence $D_{i}\left(\sigma_{i}\left(x^{k}\right), x_{i}^{k+1}, \delta\right) \geq 0$ by (2), contradicting $x^{k+1} \triangleright_{i} x^{k}$.

An AA game satisfies the strict strategic substitutes condition if

$$
D_{i}\left(s_{i}, x_{i}, \delta\right) \geq 0 \Rightarrow D_{i}\left(s_{i}-\Delta, x_{i}, \delta\right)>0
$$


for all $i \in N$ and all admissible $s_{i}, x_{i}$, and $\delta, \Delta>0$.

Theorem 2. No AA game with strict strategic substitutes admits a Cournot cycle.

For mysterious reasons, our third condition has no commonly accepted name although it is widely used in the literature (usually in a cardinal form). Kukushkin (2000b) suggested the term "dual strategic substitutes" because both (3) and (4) mean that $\operatorname{sign}\left(D_{i}\right)$ decreases along the coordinate axes in the coordinates $\langle$ total of all strategies, own strategy $\rangle$ (which are as natural as $\left\langle s_{i}, x_{i}\right\rangle$ used in this paper), while (2) means that $\operatorname{sign}\left(D_{i}\right)$ increases along the first axis.

$$
D_{i}\left(s_{i}, x_{i}, \delta\right) \geq 0 \Rightarrow D_{i}\left(s_{i}+\Delta, x_{i}-\Delta, \delta\right)>0
$$

for all $i \in N$ and all admissible $s_{i}, x_{i}$, and $\delta, \Delta>0$.

Remark. If the game is not regular, there may be no admissible $\Delta$ at all in (4), making the condition vacuous.

An AA game is symmetric if all $X_{i}$ are identical and all utilities are "isomorphic": $u_{i}(x)=V\left(\sigma_{i}(x), x_{i}\right)$ (with the same $V$ for all $\left.i \in N\right)$.

Theorem 3. No symmetric regular AA game satisfying (4) admits a Cournot cycle.

\section{Proof of Theorem 2}

Lemma 4.1. If $V_{i}\left(s_{i}, x_{i}\right)$ satisfies (3), $s_{i}^{\prime}<s_{i}^{\prime \prime}, x_{i}^{\prime} \in R_{i}\left(s_{i}^{\prime}\right)$, and $x_{i}^{\prime \prime} \in R_{i}\left(s_{i}^{\prime \prime}\right)$, then $x_{i}^{\prime} \geq x_{i}^{\prime \prime}$.

Proof. Since $x_{i}^{\prime \prime} \in R_{i}\left(s_{i}^{\prime \prime}\right), D_{i}\left(s_{i}^{\prime \prime}, x_{i}^{\prime}, x_{i}^{\prime \prime}-x_{i}^{\prime}\right) \geq 0$. If $x_{i}^{\prime}<x_{i}^{\prime \prime}$, then $D_{i}\left(s_{i}^{\prime}, x_{i}^{\prime}, x_{i}^{\prime \prime}-x_{i}^{\prime}\right)>0$ by (3), which contradicts $x_{i}^{\prime} \in R_{i}\left(s_{i}^{\prime}\right)$.

For each $i \in N, x_{i} \in X_{i}, x \in X$, and $t \in T$, we define:

$$
\begin{gathered}
Z_{i}\left(x_{i}, t\right)=\left\{z \in X \mid \quad z_{i} \in R_{i}\left(\sigma_{i}(z)\right) \& z_{i} \leq x_{i} \& \tau(z) \geq t\right\} ; \\
\Xi_{i}\left(x_{i}, t\right)=\left\{z_{i} \in X_{i} \mid \quad \exists z_{-i}\left[\left(z_{-i}, z_{i}\right) \in Z_{i}\left(x_{i}, t\right)\right]\right\} ; \\
\xi_{i}\left(x_{i}, t\right)=\max \Xi_{i}\left(x_{i}, t\right) \quad\left(\text { if } \Xi_{i}\left(x_{i}, t\right)=\emptyset, \text { then } \xi_{i}\left(x_{i}, t\right)=\min X_{i}\right) ; \\
\xi(x, t)=\left\langle\xi_{i}\left(x_{i}, t\right)\right\rangle_{i \in N} \in X .
\end{gathered}
$$


An inequality $\xi(y, t) \geq \xi(x, t)$ is understood coordinate-wise; $\xi(y, t)>\xi(x, t)$ means $\xi(y, t) \geq \xi(x, t)$ but not $\xi(x, t) \geq \xi(y, t)$ (Pareto dominance).

While following the proofs of the lemmas below, the reader might find it helpful to study on pictures how the graph of a decreasing mapping, $R_{i}\left(s_{i}\right)$, can intersect the "east-south-eastern" corners cut off by the inequalities in the definition of $Z_{i}\left(x_{i}, t\right)$ from the plane with coordinates $\left\langle s_{i}, z_{i}\right\rangle$ [ taking into account that $\tau(z)=s_{i}+z_{i}$ ]; there are too many possible configurations to be printed here. As to the meaning of the whole construction, I can only quote Kukushkin (1994): "it remains somewhat a mystery why it works at all."

Lemma 4.2. These statements hold for every $i \in N, t, t^{\prime}, t^{\prime \prime} \in T$, and $x_{i}, x_{i}^{\prime}, x_{i}^{\prime \prime} \in X_{i}$ :

4.2.1. $x_{i} \geq \xi_{i}\left(x_{i}, t\right)$;

4.2.2. $x_{i}^{\prime \prime} \geq x_{i}^{\prime} \Rightarrow \xi_{i}\left(x_{i}^{\prime \prime}, t\right) \geq \xi_{i}\left(x_{i}^{\prime}, t\right)$;

4.2.3. $t^{\prime \prime} \geq t^{\prime} \Rightarrow \xi_{i}\left(x_{i}, t^{\prime \prime}\right) \leq \xi_{i}\left(x_{i}, t^{\prime}\right)$;

4.2.4. $\xi_{i}\left(x_{i}^{\prime \prime}, t\right)>\xi_{i}\left(x_{i}^{\prime}, t\right) \Rightarrow \xi_{i}\left(x_{i}^{\prime \prime}, t\right)>x_{i}^{\prime}$;

4.2.5. $t^{\prime \prime} \geq t^{\prime} \Rightarrow \xi_{i}\left(\xi_{i}\left(x_{i}, t^{\prime}\right), t^{\prime \prime}\right)=\xi_{i}\left(x_{i}, t^{\prime \prime}\right)$;

4.2.6. $\left[\xi_{i}\left(x_{i}^{\prime \prime}, t^{\prime}\right) \geq \xi_{i}\left(x_{i}^{\prime}, t^{\prime}\right) \& t^{\prime \prime} \geq t^{\prime}\right] \Rightarrow \xi_{i}\left(x_{i}^{\prime \prime}, t^{\prime \prime}\right) \geq \xi_{i}\left(x_{i}^{\prime}, t^{\prime \prime}\right)$.

Proof. The statement [4.2.1] immediately follows from the definition; [4.2.2], from $Z_{i}\left(x_{i}^{\prime}, t\right) \subseteq Z_{i}\left(x_{i}^{\prime \prime}, t\right) ;[4.2 .3]$, from $Z_{i}\left(x_{i}, t^{\prime \prime}\right) \subseteq Z_{i}\left(x_{i}, t^{\prime}\right)$. The left hand side of [4.2.4] implies the existence of $z \in Z_{i}\left(x_{i}^{\prime \prime}, t\right)$ with $z_{i}=\xi_{i}\left(x_{i}^{\prime \prime}, t\right)$; now if $x_{i}^{\prime} \geq \xi_{i}\left(x_{i}^{\prime \prime}, t\right)$, then $z \in Z_{i}\left(x_{i}^{\prime}, t\right)$, hence $\xi_{i}\left(x_{i}^{\prime}, t\right) \geq \xi_{i}\left(x_{i}^{\prime \prime}, t\right)$, contradicting the assumption. Turning to [4.2.5], we notice that $\xi_{i}\left(\xi_{i}\left(x_{i}, t^{\prime}\right), t^{\prime \prime}\right) \leq \xi_{i}\left(x_{i}, t^{\prime \prime}\right)$ by [4.2.1] and [4.2.2]. To prove the opposite inequality, we assume the contrary: $\xi_{i}\left(\xi_{i}\left(x_{i}, t^{\prime}\right), t^{\prime \prime}\right)<\xi_{i}\left(x_{i}, t^{\prime \prime}\right)$; then $\xi_{i}\left(x_{i}, t^{\prime}\right)<\xi_{i}\left(x_{i}, t^{\prime \prime}\right)$ by [4.2.4], which contradicts [4.2.3]. Finally, [4.2.6] immediately follows from [4.2.5] and [4.2.2].

Now we define these three binary relations on $X$ :

$$
\begin{gathered}
y \succ^{\prime} x \Longleftrightarrow \exists t^{*} \geq \max \{\tau(x), \tau(y)\}\left[\xi\left(y, t^{*}\right)>\xi\left(x, t^{*}\right)\right] ; \\
y \succ^{\prime \prime} x \Longleftrightarrow[\tau(y)<\tau(x) \& \xi(y, \tau(x)) \geq \xi(x, \tau(x))] ; \\
y \succ x \Longleftrightarrow\left[y \succ^{\prime} x \text { or } y \succ^{\prime \prime} x\right] .
\end{gathered}
$$

The relation $\succ$ is obviously irreflexive. 
Lemma 4.3. The relation $\succ$ is transitive.

Proof. With every pair $y, x \in X$ such that $y \succ x$, we associate $\tau^{*}(y, x) \in T$ such that $\tau^{*}(y, x) \geq \max \{\tau(x), \tau(y)\}, \xi\left(y, \tau^{*}(y, x)\right)>\xi\left(x, \tau^{*}(y, x)\right)$ if $y \succ^{\prime} x$, and $\tau^{*}(y, x)=$ $\tau(x)$ if $y \succ^{\prime \prime} x$; in the latter case, $\xi\left(y, \tau^{*}(y, x)\right) \geq \xi\left(x, \tau^{*}(y, x)\right)$. Now, having $z \succ y \succ x$, we define $t^{*}=\max \left\{\tau^{*}(y, x), \tau^{*}(z, y)\right\}$; by Lemma $4.2, \xi\left(z, t^{*}\right) \geq \xi\left(y, t^{*}\right) \geq \xi\left(x, t^{*}\right)$. If $t^{*}$ can be associated with a $\succ^{\prime}$ relation, one of the inequalities is strict, hence $\xi\left(z, t^{*}\right)>$ $\xi\left(x, t^{*}\right)$, hence $z \succ^{\prime} x$. Otherwise, $y \succ^{\prime \prime} x, t^{*}=\tau(x)>\tau(y)$, and $\tau(x)>\tau^{*}(z, y) \geq \tau(z)$; now $\xi(z, \tau(x)) \geq \xi(x, \tau(x))$ implies $z \succ^{\prime \prime} x$.

Lemma 4.4. If $y \triangleright x$, then $y \succ x$.

Proof. Let $y \triangleright_{i} x$; we have to consider two alternatives.

Let $y_{i}>x_{i}$; we define $t^{*}=\tau(y)>\tau(x)$. We have $y \in Z_{i}\left(y_{i}, t^{*}\right)$, hence $\xi_{i}\left(y_{i}, t^{*}\right)=$ $y_{i}>x_{i} \geq \xi_{i}\left(x_{i}, t^{*}\right)$; on the other hand, for $j \neq i$, we have $\xi_{j}\left(y_{j}, t^{*}\right)=\xi_{j}\left(x_{j}, t^{*}\right)$ since $y_{j}=x_{j}$. Thus $\xi\left(y, t^{*}\right)>\xi\left(x, t^{*}\right)$, hence $y \succ^{\prime} x$.

Now let $y_{i}<x_{i}$; then $\tau(y)<\tau(x)$. For every $j \neq i$, we have $\xi_{j}\left(y_{j}, \tau(x)\right)=$ $\xi_{j}\left(x_{j}, \tau(x)\right)$ since $y_{j}=x_{j}$. Suppose $\xi_{i}\left(y_{i}, \tau(x)\right)<\xi_{i}\left(x_{i}, \tau(x)\right)$ and pick $z \in Z_{i}\left(x_{i}, \tau(x)\right)$ for which $z_{i}=\xi_{i}\left(x_{i}, \tau(x)\right)$; then $x_{i} \geq z_{i}>y_{i}$ by [4.2.1] and [4.2.4]. Since $\tau(z) \geq \tau(x)$, we have $\sigma_{i}(z) \geq \sigma_{i}(x)=\sigma_{i}(y)$, but $\sigma_{i}(z)=\sigma_{i}(x)$ is impossible because of $x_{i} \notin$ $R_{i}\left(\sigma_{i}(x)\right)$. Now $\sigma_{i}(z)>\sigma_{i}(x), z_{i}>y_{i}, z_{i} \in R_{i}\left(\sigma_{i}(z)\right)$, and $y_{i} \in R_{i}\left(\sigma_{i}(x)\right)$ contradict Lemma 4.1. Therefore, $\xi(y, \tau(x)) \geq \xi(x, \tau(x))$, hence $y t^{\prime \prime} x$.

The impossibility of a Cournot cycle now immediately follows from Lemmas 4.3 and 4.4. Theorem 2 is proved.

\section{$5 \quad$ Proof of Theorem 3}

Considering symmetric games, we denote $Y=X_{i}$ for all $i \in N$ and omit the subscript $i$ at $V, S, R, D$, etc.

Lemma 5.1. If $V(s, \xi)$ satisfies (4), $s^{\prime}<s^{\prime \prime}, \xi^{\prime} \in R\left(s^{\prime}\right)$, and $\xi^{\prime \prime} \in R\left(s^{\prime \prime}\right)$, then $s^{\prime}+\xi^{\prime} \leq s^{\prime \prime}+\xi^{\prime \prime}$. 
Proof. Supposing the contrary, we denote $\Delta=s^{\prime \prime}-s^{\prime}>0, \delta=\xi^{\prime}-\xi^{\prime \prime}-\Delta>0$. Since $\xi^{\prime \prime} \in R\left(s^{\prime \prime}\right), D\left(s^{\prime \prime}, \xi^{\prime \prime}, \delta\right) \leq 0$; (4) then implies $D\left(s^{\prime}, \xi^{\prime \prime}+\Delta, \delta\right)<0$, hence $V\left(s^{\prime}, \xi^{\prime \prime}+\Delta\left[=\xi^{\prime}-\delta\right]\right)>V\left(s^{\prime}, \xi^{\prime}\right)$, which contradicts $\xi^{\prime} \in R\left(s^{\prime}\right)$.

For every $x \in X$ and $m \in Y$, we define:

$$
\begin{gathered}
\mu(x)=\max _{i \in N} x_{i} ; \quad M(x)=\underset{i \in N}{\operatorname{Argmax}} x_{i} ; \\
\Psi(m)=\{t \in T \mid \quad \forall s \in S[s+m \geq t \Rightarrow[m \in R(s) \text { or } \forall \xi \in R(s)(s+\xi \geq t)]]\} ; \\
\Phi(x)=\{t \in \Psi(\mu(x)) \mid \quad t \leq \tau(x)\} ; \quad \varphi(x)=\max \Phi(x)
\end{gathered}
$$

[note that $\min T \in \Phi(x) \neq \emptyset$ and $\varphi(x) \leq \tau(x)$ for every $x \in X$ by definition];

$$
\begin{gathered}
y \succeq x \Longleftrightarrow[[\varphi(y)>\varphi(x)] \text { or }[\varphi(y)=\varphi(x) \& \mu(y)<\mu(x)] \text { or } \\
[\varphi(y)=\varphi(x) \& \mu(y)=\mu(x) \& M(y) \subseteq M(x)]] ; \\
y \succ x \Longleftrightarrow[[\varphi(y)>\varphi(x)] \text { or }[\varphi(y)=\varphi(x) \& \mu(y)<\mu(x)] \text { or } \\
[\varphi(y)=\varphi(x) \& \mu(y)=\mu(x) \& M(y) \subset M(x)]] .
\end{gathered}
$$

Obviously, $\succeq$ is transitive and $\succ$ is the asymmetric component of $\succeq(y \succ x \Longleftrightarrow$ $[y \succeq x$ and not $x \succeq y]$ ).

The above constructions, unlike those from the previous section, admit an intuitive explanation. $t \in \Psi(m)$ means that any strategy profile which sums up to $t$ or more and contains no component greater than $m$ cannot sum up to less than $t$ after any best response improvement; $\varphi(x)$ is thus "the greatest self-supporting level below $x . "$ If $\mu$ does not increase (at a step of the process), whatever was self-supporting remains self-supporting, so $\varphi$ cannot decrease (Lemma 5.2). When $\mu$ increases, we cannot be so sure about the previously self-supporting levels; fortunately, the new sum is then self-supporting itself (Lemma 5.3).

Lemma 5.2. If $y \triangleright_{i} x$ and $y_{i} \leq \mu(x)$, then $\varphi(y) \geq \varphi(x)$.

Proof. Let $s^{0}$ be $\min \{s \in S \mid s+\mu(x) \geq \varphi(x)\}$ [actually, either $s^{0}=\varphi(x)-\mu(x)$ or $\left.s^{0}=\min S\right]$. If, for $t=\varphi(x)$ and $s=s^{0}$, the second alternative in the definition of $\Psi(\mu(x))$ above holds, then, by Lemma 5.1, $s+\xi \geq \varphi(x)$ for all $s \geq s^{0}$ and $\xi \in R(s)$, in particular, for $s=\sigma_{i}(x)$ and $\xi=y_{i}$; therefore, $\tau(y) \geq \varphi(x)$ and, since 
$\mu(y) \leq \mu(x), \varphi(x) \in \Phi(y)$, hence $\varphi(x) \leq \varphi(y)$. If $\mu(x) \in R\left(s^{0}\right)$, then, by Lemma 5.1, $s+\xi \geq s^{0}+\mu(x)$ for all $s>s^{0}$ and $\xi \in R(s)$, hence $\varphi(x)=s^{0}+\mu(x)$; since $x_{i} \notin R\left(\sigma_{i}(x)\right), \sigma_{i}(x)>s^{0}$, hence $\tau(y) \geq \varphi(x)$, hence $\varphi(x) \leq \varphi(y)$ exactly as in the previous case.

Lemma 5.3. If $y \triangleright_{i} x$ and $y_{i} \geq \mu(x)$, then $\varphi(y)>\varphi(x)$.

Proof. We obviously have $\mu(y)=y_{i}>x_{i}$, hence $\tau(y)>\tau(x)$. Furthermore, $y_{i} \in$ $R\left(\sigma_{i}(y)\right)$ implies, by Lemma 5.1, that $s+\xi \geq \tau(y)$ for all $s>\sigma_{i}(y)$ and $\xi \in R(s)$, hence $\tau(y) \in \Phi(y)$, hence $\varphi(y)=\tau(y)>\tau(x) \geq \varphi(x)$.

Lemma 5.4. Let $y \triangleright_{i} x$; then $y \succeq x$. If $i \in M(x)$, then $y \succ x$. If $x \succeq y$, then $M(y)=M(x)$.

Proof. Let $i \in M(x)$; if $y_{i}>x_{i}$, then $y_{i}>\mu(x)$, hence $y \succ x$ by Lemma 5.3. Let $y_{i}<x_{i}$; then $\varphi(y) \geq \varphi(x)$ by Lemma 5.2. If $M(x)=\{i\}$, then $\mu(y)<\mu(x)$, hence $y \succ x$. If $\{i\} \subset M(x)$, then $\mu(y)=\mu(x)$ and $M(y) \subset M(x)$; again, $y \succ x$.

Let $i \notin M(x)$; if $y_{i} \geq \mu(x)$, then Lemma 5.3 implies $y \succ x$; otherwise, we have $\mu(y)=\mu(x), M(y)=M(x)$, and $\varphi(y) \geq \varphi(x)$ by Lemma 5.2 .

Supposing the existence of symmetric regular AA games satisfying (4), but admitting Cournot cycles, we pick such a game with a minimal number of players. Let $x^{0}, x^{1}, \ldots, x^{m}=x^{0}$ be a Cournot cycle. If $x^{k+1} \succ x^{k}$ for some $k$, we have a contradiction with the transitivity of $\succeq$. Otherwise, Lemma 5.4 implies $M\left(x^{0}\right)=M\left(x^{1}\right)=$ $\cdots=M\left(x^{m-1}\right)=N^{*}$ and $x^{k+1} \triangleright_{i} x^{k}$ for $i \in N^{*}$ never takes place, i.e., $x_{i}^{k}$ for $i \in N^{*}$ does not depend on $k$. The last statement implies that the reduced game with $N \backslash N^{*}$ as the set of players also admits (the same) Cournot cycle, which contradicts the supposed minimality of $N$. Theorem 3 is proved.

\section{$6 \quad$ Numeric and Order Potentials}

In this section, we consider arbitrary finite games. The best response correspondence $R_{i}(\cdot)(i \in N)$ will be perceived as defined on $X_{-i}$ :

$$
R_{i}\left(x_{-i}\right)=\underset{x_{i} \in X_{i}}{\operatorname{Argmax}} u_{i}\left(x_{i}, x_{-i}\right) .
$$


Besides the Cournot (best response) relation defined at the start of Section 2, a large number of other relations on $X$ are worth being considered; e.g., $y \triangleright x \Longleftrightarrow \exists i \in$ $N\left[y_{-i}=x_{-i} \& u_{i}(y)>u_{i}(x)\right]$ (Monderer and Shapley's unilateral improvement relation), or $y \triangleright x \Longleftrightarrow \forall i \in N\left[y_{i}=x_{i} \in R_{i}\left(x_{-i}\right)\right.$ or $\left.x_{i} \notin R_{i}\left(x_{-i}\right) \ni y_{i}\right]$ (a version of simultaneous best response "improvement"), or $y \triangleright x \Longleftrightarrow \exists I \in 2^{N} \backslash\{\emptyset\}\left[y_{-I}=\right.$ $\left.x_{-I} \& \forall i \in I\left[u_{i}(y)>u_{i}(x)\right]\right]$ (a version of coalition improvement), to name just a few. Statements of highest generality are independent of exactly what is meant by $\triangleright$.

Thus, let $\triangleright$ be a binary relation on a finite set $X$. An improvement path (for $\triangleright$ ) is a sequence $\left\{x^{k}\right\}_{k=0,1, \ldots}$ such that $x^{k+1} \triangleright x^{k}$ whenever $x^{k+1}$ is defined. If $x^{k}$ is defined exactly for $k=0, \ldots, m$, we call $m \geq 0$ the length of the path (thus a path consisting of a single point has the length 0 ). The relation $\triangleright$ has the finite improvement path $(F I P)$ property if there exists no infinite improvement path; the property is obviously equivalent to the absence of improvement cycles, i.e., to the acyclicity of the relation. The relation $\triangleright$ has the weak FIP property if every $x \in X$ is connected to a maximizer for $\triangleright$ with an improvement path, i.e., there is a finite improvement path $\left\{x^{0}, \ldots, x^{m}\right\}$ $(m \geq 0)$ such that $x^{0}=x$ and $x^{m}$ is a maximizer. Clearly, FIP implies weak FIP, which, in turn, implies the existence of a maximizer for $\triangleright$.

Remark. Monderer and Shapley (1996a) introduced the term FIP for a particular relation, viz. for the individual improvement relation in a strategic game; here we apply it to an abstract binary relation. The hope is that the context will always allow us to avoid confusion; besides, the "abstract" FIP is the property of a binary relation whereas "Monderer and Shapley's" FIP is the property of a game.

We say that a function $P: X \rightarrow \mathbb{R}$ is a numeric potential for $\triangleright$ if

$$
y \triangleright x \Rightarrow P(y)>P(x)
$$

for all $y, x \in X$ (utility theorists would say that $P$ weakly represents $\triangleright$ ). An order potential for $\triangleright$ is an irreflexive and transitive binary relation $\succ$ on $X$ such that

$$
y \triangleright x \Rightarrow y \succ x
$$

for all $y, x \in X$. A weak order potential for $\triangleright$ is an irreflexive and transitive binary relation $\succ$ on $X$ such that

$$
\exists y \in X[y \triangleright x] \Rightarrow \exists z \in X[z \triangleright x \& z \succ x]
$$


for all $x \in X$. A weak numeric potential for $\triangleright$ is a function $P: X \rightarrow \mathbb{R}$ such that

$$
\exists y \in X[y \triangleright x] \Rightarrow \exists z \in X[z \triangleright x \& P(z)>P(x)]
$$

for all $x \in X$.

Proposition 6.1. For any binary relation $\triangleright$ on a finite set $X$, the following statements are equivalent:

6.1.1. $\triangleright$ admits a numeric potential;

6.1.2. $\triangleright$ admits an order potential;

6.1.3. $\triangleright$ has the FIP property.

Proof. The implications $[6.1 .1] \Rightarrow[6.1 .2]$ and $[6.1 .2] \Rightarrow[6.1 .3]$ are straightforward. To prove $[6.1 .3] \Rightarrow[6.1 .1]$, we define $p(x)=$ [the maximal length of an improvement path starting at $x](\leq \# X-1)$ and $P(x)=-p(x)$ for every $x \in X$. Now $y \triangleright x$ implies that every improvement path starting at $y$ can be extended (on the left) to a path starting at $x$; therefore, $p(x) \geq p(y)+1$, hence $P(y)>P(x)$.

Remark. Proposition 6.1 generalizes Lemma 2.5 of Monderer and Shapley (1996a).

Proposition 6.2. For any binary relation $\triangleright$ on a finite set $X$, the following statements are equivalent:

6.2.1. $\triangleright$ admits a weak numeric potential;

6.2.2. $\triangleright$ admits a weak order potential;

6.2.3. $\triangleright$ has the weak FIP property.

Proof. The implication [6.2.1] $\Rightarrow[6.2 .2]$ is obvious. To prove [6.2.2] $\Rightarrow[6.2 .3]$, we define the following rule for continuing improvement paths: if $x^{k}$ is not a maximizer, we pick $x^{k+1}$ such that $x^{k+1} \triangleright x^{k}$ and $x^{k+1} \succ x^{k}$. Now, starting with an arbitrary $x^{0}$ and applying the rule, we obtain an improvement path that cannot cycle and only stops at a maximizer for $\triangleright$; therefore, we have the weak FIP indeed. To prove [6.2.3] $\Rightarrow$ [6.2.1], we define $p(x)=$ the minimal length of an improvement path starting at $x$ and ending at a maximizer $](\leq \# X-1)$ and $P(x)=-p(x)$ for every $y, x \in X$. Now $y \triangleright x$ implies $p(x)>0$; we pick an improvement path $\left\{x^{k}\right\}_{k=0, \ldots, p(x)}$ such that $x^{0}=x$ and $x^{p(x)}$ is a maximizer, and define $z=x^{1}$. Clearly, $p(z)=p(x)-1$, hence $P(z)>P(x)$; on the other hand, $z=x^{1} \triangleright x^{0}=x$. 
Remark. There is no tradition of considering weak potentials in the literature. Both Milchtaich (1996) and Friedman and Mezzetti (2001) established the weak FIP property of some improvement relations in strategic games without producing explicit potentials.

Henceforth, we return to finite strategic games and denote $\triangleright$ the Cournot relation as defined in Section 2. The (weak) FIP property of this relation will be called (weak) FBRP property of the game, following Milchtaich (1996). As suggested by the associate editor, a numeric potential for $\triangleright$ will be called a generalized best response potential of the game.

Proposition 6.3. For any finite game, a function $P: X \rightarrow \mathbb{R}$ is a generalized best response potential if and only if for every $i \in N$ and $x_{-i} \in X_{-i}, R_{i}\left(x_{-i}\right)$ is an upper contour for the function $P\left(\cdot, x_{-i}\right)$, i.e., there exists a threshold level $w\left(x_{-i}\right) \in \mathbb{R}$ such that

$$
R_{i}\left(x_{-i}\right)=\left\{x_{i}^{\prime} \in X_{i} \mid P\left(x_{i}^{\prime}, x_{-i}\right) \geq w\left(x_{-i}\right)\right\} .
$$

Proof. If $P$ satisfies (6) and $y \triangleright x$, i.e., $y_{-i}=x_{-i}$ and $x_{i} \notin R_{i}\left(x_{-i}\right) \ni y_{i}$, then $P(y) \geq w\left(x_{-i}\right)>P(x)$, implying (5). Let $P$ satisfy (5) and $x_{-i} \in X_{-i}$; we pick $x_{i} \in$ $R_{i}\left(x_{-i}\right)$ minimizing $P\left(\cdot, x_{-i}\right)$ on $R_{i}\left(x_{-i}\right)$ and denote $w\left(x_{-i}\right)=P\left(x_{i}, x_{-i}\right)$. Whenever $y_{i} \in R_{i}\left(x_{-i}\right)$, we have $P\left(y_{i}, x_{-i}\right) \geq w\left(x_{-i}\right)$ by definition; whenever $y_{i} \notin R_{i}\left(x_{-i}\right)$, we have $\left(x_{i}, x_{-i}\right) \triangleright\left(y_{i}, x_{-i}\right)$, hence, by $(5), w\left(x_{-i}\right)=P(x)>P\left(y_{i}, x_{-i}\right)$. Now (6) is obvious.

The equality

$$
R_{i}\left(x_{-i}\right)=\underset{x_{i}^{\prime} \in X_{i}}{\operatorname{Argmax}} P\left(x_{i}^{\prime}, x_{-i}\right)
$$

suffices, but it need not hold as the example from Monderer and Shapley (1996a, p. 129) shows (in a $2 \times 2$ game, the best response and a better response amount to the same thing). The condition is much more natural when the "fictitious play" property (Monderer and Shapley, 1996b; Huang, 2002) is under consideration; there seems to be no result on the FP property of games admitting a potential in the sense of (6). The characterization of games admitting a potential satisfying (7) in terms of "best response cycles" (Voorneveld, 2000) resembles Propositions 6.1 and 6.3 only superficially: a "best response compatible path" may pass through a Nash equilibrium without noticing it. 
A property intermediate between FBRP and weak FBRP deserves attention. A finite game has the restricted FBRP property if there exist nonempty $R_{i}^{\prime}\left(x_{-i}\right) \subseteq$ $R_{i}\left(x_{-i}\right)$, for all $i \in N$ and $x_{-i} \in X_{-i}$, such that the relation $y \triangleright^{*} x \Longleftrightarrow \exists i \in$ $N\left[y_{-i}=x_{-i} \& u_{i}(y)>u_{i}(x) \& y_{i} \in R_{i}^{\prime}\left(x_{-i}\right)\right]$ is acyclic (i.e., has the "abstract" FIP property). Clearly, FBRP $\Rightarrow$ restricted FBRP $\Rightarrow$ weak FBRP.

Remark. FBRP itself can be considered a restricted version of Monderer and Shapley's FIP; however, it hardly makes sense to formalize the idea here.

A restricted best response potential is an irreflexive and transitive binary relation $\succ$ on $X$ such that

$$
\exists y \in X\left[y \triangleright_{i} x\right] \Rightarrow \exists z \in X\left[z \triangleright_{i} x \& z \succ x\right]
$$

for all $x \in X$ and $i \in N$. Dubey et al. (2002) defined a "pseudopotential" as a function $P: X \rightarrow \mathbb{R}$ such that

$$
\underset{x_{i}^{\prime} \in X_{i}}{\operatorname{Argmax}} P\left(x_{i}^{\prime}, x_{-i}\right) \subseteq R_{i}\left(x_{-i}\right)
$$

for all $i \in N$ and $x_{-i} \in X_{-i}$ (their continuity condition is satisfied automatically in a finite game); their Theorem 3 states, in particular, that every finite game admitting a pseudopotential has a Nash equilibrium. Proposition 6.3 above immediately implies that every generalized best response potential is a pseudopotential.

Proposition 6.4. For any finite game $\Gamma$, these statements are equivalent:

6.4.1. $\Gamma$ admits a restricted best response potential;

6.4.2. $\Gamma$ admits a pseudopotential in the sense of (8);

6.4.3. $\Gamma$ has the restricted FBRP property.

Proof. If [6.4.1] holds, we define $y \triangleright_{i} x \Longleftrightarrow y \triangleright_{i} x \& y \succ x$ and $y \bowtie x \Longleftrightarrow \exists i \in$ $N\left[y \bowtie_{i} x\right]$. Obviously, $\succ$ is an order potential for $\bowtie$; Proposition 6.1 implies the existence of a numeric potential $P$ for $\bowtie$. Let us show that $P$ satisfies (8). Suppose the contrary: $x_{i} \in \operatorname{Argmax}_{x_{i}^{\prime} \in X_{i}} P\left(x_{i}^{\prime}, x_{-i}\right)$, but $x_{i} \notin R_{i}\left(x_{-i}\right)$. Picking $y_{i} \in R_{i}\left(x_{-i}\right)$, we see that $\left(y_{i}, x_{-i}\right) \triangleright_{i} x$, hence there is $z \in X$ such that $z \triangleright_{i} x$, hence $P(z)>P(x)$; clearly, $z_{-i}=x_{-i}$, which contradicts the choice of $x_{i}$.

If [6.4.2] holds, we define $R_{i}^{\prime}\left(x_{-i}\right)=\operatorname{Argmax}_{x_{i}^{\prime} \in X_{i}} P\left(x_{i}^{\prime}, x_{-i}\right)\left[\subseteq R_{i}\left(x_{-i}\right)\right.$ by (8)]. Obviously, $P$ is a numeric potential for $\triangleright^{*}$, hence $\triangleright^{*}$ is acyclic, i.e., [6.4.3] holds. 
If [6.4.3] holds, we denote $\succ$ an order potential for $\triangleright^{*}$, existing by Proposition 6.1. It is easy to see that $\succ$ is a restricted best response potential.

Remark. The proof of $[6.4 .2] \Rightarrow[6.4 .3]$ also shows that every pseudopotential is a weak numeric best response potential.

Proposition 6.5. A finite two person game has the weak FBRP property if and only if it has the restricted FBRP property.

Proof. The sufficiency holds for every finite game. Let a game with $\# N=2$ have the weak FBRP property. In the light of Proposition 6.4, it is sufficient to produce a pseudopotential. We use the construction from the proof of Proposition 6.2 with some modification utilizing the two person assumption.

For $x \in X$, we define $I(x)=\left\{i \in N \mid x_{i} \notin R_{i}\left(x_{-i}\right)\right\}$, and denote $X^{*}=\{x \in$ $X \mid I(x)=N\}$. Since a best response cannot be improved upon, $y \triangleright_{i} x \Rightarrow i \notin I(y)$; therefore, $x^{k} \notin X^{*}$ for all Cournot paths $x^{0}, x^{1}, \ldots$ and all $k>0$. Now we define $p(x)$ for $x \in X \backslash X^{*}$ exactly as in Proposition 6.2 ; for $x \in X^{*}$, we define $p(x)=$ $\max _{i \in N} \min _{y \triangleright i} x p(y)+1$; finally, $P(x)=-p(x)$ for all $x \in X$. It is easy to see that $P$ satisfies the equation

$$
P(x)=\min _{i \in I(x)} \max _{y \triangleright_{i} x} P(y)-1
$$

(since $\# N=2$, the minimum in (9) is essential for $x \in X^{*}$ only) with the boundary condition $P(x)=0$ for Nash equilibria.

Equation (9) ensures that $P$ is a pseudopotential (even regardless of $\# N=2$ ): Let $i \in N, x_{-i} \in X_{-i}$, and $x_{i} \notin R_{i}\left(x_{-i}\right)$; then $i \in I(x)$. Let $z \triangleright_{i} x$ maximize $P(y)$ over all $y \triangleright_{i} x$; it follows immediately from $(9)$ that $P(z)>P(x)$. Therefore, $x_{i}$ does not maximize $P\left(\cdot, x_{-i}\right)$.

Remark. A weak numeric best response potential need not be a pseudopotential, even in a two person game.

To summarize, both numeric and order approaches to finite potential games, including best response potentials, coalition improvement potentials (see, e.g., Holzman and Law-Yone, 1997), etc., are theoretically equivalent; the choice, in each case, should be based on convenience. The definition of the numeric potential in the proof of Proposition 6.1 is easily converted into a dynamic programming algorithm; for two or three person games with not too many strategies, the algorithm only needs a pen 
and a piece of paper to work (see Section 7.10). The same is true for the weak numeric potential from Proposition 6.2. However, such algorithms could hardly be used to establish that every game from an infinite class admits a potential. The proofs of the theorems of this paper demonstrate various ways to do so; in none of them a potential emerges as a numeric function, even though Propositions 6.1 and 6.3 imply that every game satisfying the conditions of any of the theorems admits a numeric potential in the sense of (6).

For binary relations on infinite sets, only two implications, [6.1.1] $\Rightarrow[6.1 .2] \Leftarrow$ [6.1.3], remain of Proposition 6.1. One inevitably gets a feeling that the FIP property is "too good to be true." Assuming $X$ a separable metric space, Kukushkin (2000a) considered improvement paths parameterized by ordinal (generally, transfinite) numbers; the absence of improvement cycles is then equivalent to the existence of an order potential (as defined in Kukushkin, 1999) and to the "countable improvement path" property, i.e., to the impossibility of an uncountable improvement path, which, in turn, implies that every improvement path in a compact space eventually reaches an equilibrium. Analogues of Theorems 1-3 hold in that setting; even the constructions proving Theorems 2 and 3 remain essentially the same (only more technical details is needed). Certainly, one does not have to accept the theory, but there seems to be no alternative.

\section{Concluding Remarks}

7.1. Although the subtraction of utilities is used in (1), the properties (2-4) are purely ordinal, i.e., invariant under any strictly increasing transformation of the utility function. Accordingly, this paper bears no relation whatsoever to the literature on cardinal notions of a potential (including a considerable part of Monderer and Shapley, 1996a).

7.2. Exact analogues of Theorems 1 and 2 are valid for games with separable (not necessarily additive) aggregation; the proofs are virtually the same as above because subtraction is never used in either. Section 7 of Dubey et al. (2002) suggests that even separability need not be necessary. Without any assumption on aggregation, both theorems are definitely wrong.

As to Theorem 3, subtraction is essential in Lemma 5.1; although the condition (4) 
can be reformulated without subtraction, it can prove meaningless. However, assuming that each player is only affected by a separable (and symmetric), but not necessarily additive, aggregate, and postulating the "cumulative monotonicity" (Lemma 5.1) of the best replies, one can then prove the impossibility of a Cournot cycle in the same way as in Section 5 (again, there is no subtraction there).

7.3. It remains unclear whether Theorem 2 holds under the nonstrict strategic substitutes condition, i.e., under (3) with the nonstrict inequality. As to Theorem 3, it is definitely wrong under the "nonstrict dual strategic substitutes" condition.

Consider a symmetric regular AA game with $N=\{1,2\}, X_{1}=X_{2}=\{0,1,2,3\}$, and utilities defined by the following matrix $V$ (with the partner's choice, " $s_{i}$," on the abscissae axis, directed from left to right, and the player's own choice, " $x_{i}$," on the ordinates axis, directed upwards):

$\begin{array}{llll}0 & 2 & 1 & 1 \\ 1 & 1 & 2 & 2 \\ 2 & 2 & 1 & 1 \\ 1 & 1 & 2 & 0\end{array}$

The nonstrict version of (4) is easily checked. We depict the utilities matrices, again assuming that the axes go from left to right and from bottom to top, thus breaking with a venerated tradition to have rows numbered in the opposite order; however, we follow the tradition in treating the vertical axis as belonging to player 1 and the horizontal one as belonging to player 2 :

$$
\begin{array}{llll}
(0,0) & \underline{(2,1)} & \underline{(1,2)} & (1,1) \\
(1,2) & (1,1) & (2,2) & (2,1) \\
(2,1) & (2,2) & (1,1) & (1,2) \\
(1,1) & \underline{(1,2)} & \underline{(2,1)} & (0,0)
\end{array}
$$

Now a Cournot cycle is easy to see (underlined above).

7.4. It is easy to see that, if $R_{i}(\cdot)$ is replaced (naturally, starting from Section 2) with $R_{i}^{\prime}(\cdot) \subseteq R_{i}(\cdot)$ satisfying the statement of Lemma 4.1 [or Lemma 5.1], all further arguments in Section 4 [or 5] remain valid. The nonstrict version of (3) [or (4)] implies the existence of a singleton selection from the best response correspondence with this property: the greatest best response will do. Therefore, the nonstrict strategic substitutes condition in a finite game with additive aggregation ensures, at least, the 
restricted FBRP property. The same is true for (4) with symmetry. (The point of the example in Section 7.3 was that player 1 used a selection violating the cumulative monotonicity.)

7.5. An equilibrium exists under (4), even in the nonstrict version, in quasisymmetric regular games, i.e., when $V$ is the same for all $i \in N$, but $X_{i}$ may differ (Kukushkin, 2000b); it remains unclear whether Cournot cycles can emerge in such games (even the weak FBRP property is by no means obvious).

7.6. The absence of Cournot cycles does not imply Monderer and Shapley's FIP property:

$$
\begin{array}{lll}
(0,0) & \frac{(1,3)}{(3,4)} & \frac{(3,3)}{(4,0) .} \\
\frac{(3,1)}{(4,3)} & \underline{(0,4)} & \underline{(0,0)}
\end{array}
$$

This is even a game with strictly increasing differences, but it admits an improvement cycle (underlined above). Such examples have been known to researchers in the field for quite some time (e.g., Sela, 1992), but seem to have never been published.

7.7. When $\# N>2$, Proposition 6.5 works only one way. Let us consider a three person $2 \times 2 \times 2$ game (where player 1 chooses rows, player 2 columns, and player 3 matrices):

$$
\left[\begin{array}{ll}
(0,1,0) & (1,0,1) \\
(1,0,1) & (0,1,1)
\end{array}\right] \quad\left[\begin{array}{ll}
(1,1,1) & (0,0,0) \\
(0,0,0) & (0,0,0)
\end{array}\right] .
$$

The "northwestern" corner of the right matrix is a unique Nash equilibrium; the weak FBRP is obvious. Nevertheless, there is no restricted FBRP because the unique best responses of players 1 and 2 in the left matrix form a cycle.

The example clarifies the difference between the two properties. Under the restricted FBRP, we can impose independent restrictions on each player's actions ensuring that an equilibrium will be reached eventually. Under the weak FBRP, cooperation between the players may be needed: if player 1 always moves from the northwestern corner of the left matrix before player 3, the equilibrium will never be reached. (Explicit cooperation can be replaced with a stochastic choice of the player to move at each step, as in Milchtaich, 1996, or Friedman and Mezzetti, 2001.)

7.8. Quite a few authors considered processes where, at each step, all players make their best response improvements simultaneously: an endomorphism $X \rightarrow X$ (if 
the uniqueness of the best responses is assumed additionally) may seem a simpler mathematical object than the Cournot relation $\triangleright$. However, the behaviour of the players in such processes is distinctly less rational than in those considered here: In this paper, there is one active player at each step, who increases his utility level; other players may suffer, but, at least, they do not contribute to their suffering. In a process with simultaneous moves, the players may participate in diminishing their own utilities. It is not surprising, therefore, that our assumptions do not exclude the cycling of such processes. On the other hand, the claim of Rand (1978) to have shown the possibility of rather complicated simultaneous best response improvement dynamics in a Cournot duopoly with zero costs (where the nonstrict version of (4) holds) is unfounded: the reaction functions there violate the cumulative monotonicity property, established in such models by McManus (1962).

7.9. There are two quite recent papers on improvement dynamics in games with additive aggregation. It seems worthwhile to compare them with this paper.

Dindoš and Mezzetti (2001) study the convergence to the set of Nash equilibria of some stochastic better reply processes; in the case of a finite game, this amounts to studying the weak FIP property. The authors concentrate on continuous games, however, relying on fashionable analytical techniques and boldly piling up assumptions. Considering discretization, they assume that any discrete model must contain the equilibria of the continuous model (where they exist by concavity); thus, our regular games would be inadmissible in most cases. This paper concentrates on finite games, in order to investigate the underlying combinatorics.

Dubey et al. (2002) is much closer in spirit to this paper. Its main advantage is that their scheme (a first version of which was developed by Huang, 2002, for somewhat different purposes) treats strategic complements and substitutes symmetrically. This should be contrasted with the fact that the proofs of Theorems 1 and 2 here have literally nothing in common despite considerable similarity in formulations [the asymmetry could be vindicated, though, were it proven that Theorem 2 is wrong under nonstrict (3) ]. Second, they can treat some nonseparable forms of aggregation which avoid this approach; for instance, the sum of all partners' strategies (in each player's utility) can be replaced with the sum of the strategies of neighbours, provided the reciprocity condition holds: if $i$ is $j$ 's neighbour, then $j$ is $i$ 's neighbour.

The weakest point of the scheme is its all but absolute refusal to have anything 
to do with multiple responses; accordingly, the main results are about "pseudopotentials" rather than potentials. It cannot also be applied to general (nonadditive) separable aggregates covered by this paper. Apparently, we should hope for a synthesis in the future.

7.10. The three theorems of this paper do not exhaust all possible conditions for the absence of Cournot cycles. Let us consider the following bimatrix game:

$$
\begin{array}{lllll}
d & (0,0) & (2,1) & (0,1) & (1,2) \\
c & (1,2) & (1,1) & (1,1) & (0,0) \\
b & (1,0) & (1,1) & (1,1) & (0,0) \\
a & (2,1) & (0,0) & (0,0) & (1,2)
\end{array}
$$

$$
\begin{array}{llll}
A & B & C & D
\end{array}
$$

It has even Monderer and Shapley's FIP property: the numeric potential defined in the proof of Proposition 6.1 looks as follows:

$$
\begin{array}{ccccc}
d & -5 & -1 & -4 & 0 \\
c & -2 & -3 & -3 & -4 \\
b & -3 & -2 & 0 & -3 \\
a & -1 & -4 & -4 & 0 \\
& A & B & C & D
\end{array}
$$

On the other hand, the game cannot be made symmetric by any reshuffling of the strategies and/or monotonic transformations of utilities (e.g., no strategy of player 1 participates in two distinct equilibria while $D$ of player 2 does), so Theorem 3 cannot be applicable. Were Theorem 2 applicable, the standard trick with reversing the order on one of $X_{i}$ 's would make Theorem 1 applicable too.

Let us suppose that the strategies of each player can be embedded into the real line (i.e., ordered) in such a way that (2) holds. Looking at the "southwestern" corner of the utility matrix, we see that the pairs $\langle A, B\rangle$ and $\langle a, b\rangle$ must be ordered in the same way, i.e., either $A>B$ and $a>b$, or $A<B$ and $a<b$. Similarly, in the same way must be ordered $\langle A, B\rangle$ and $\langle c, d\rangle$, as well as $\langle C, D\rangle$ and $\langle c, d\rangle$. However, when we look at the "southeastern" corner, we can see that $\langle C, D\rangle$ and $\langle a, b\rangle$ must be ordered oppositely (i.e., if $C<D$, then $a>b$; if $C>D$, then $a<b$ ). Clearly, these requirements are incompatible. 


\section{References}

Bulow, J.I., Geanakoplos, J.D., and Klemperer, P.D. (1985). "Multimarket Oligopoly: Strategic Substitutes and Complements," J. Polit. Econ. 93, 488-511.

Dindoš, M., and Mezzetti, C. (2001). "Better-Reply Dynamics and Global Convergence to Nash Equilibrium in Playing-Against-The-Sum Games," University of North Carolina (http://www.unc.edu/ ${ }^{\sim}$ mezzetti/better-rep2.pdf).

Dubey, P., Haimanko, O., and Zapechelnyuk, A. (2002). "Strategic Complements and Substitutes, and Potential Games," Monaster Center for Economic Research, Ben-Gurion University, DP \# 02-12.

Friedman, J.W., and Mezzetti, C. (2001). "Learning in Games by Random Sampling," J. Econ. Theory 98, 55-84.

Fudenberg, D., and Tirole, J. (1991). Game Theory, Cambridge, Mass.: The MIT Press.

Holzman, R. and Law-Yone, N. (1997). "Strong Equilibrium in Congestion Games," Games Econ. Behav. 21, 85-101.

Huang, Z. (2002). "Fictitious Play in Games with a Continuum of Strategies," Ph. D. Thesis, State University of New York at Stony Brook, Department of Economics.

Kukushkin, N.S. (1994). "A Fixed-Point Theorem for Decreasing Mappings," Econ. Lett. 46, 23-26.

Kukushkin, N.S. (1999). "Potential Games: A Purely Ordinal Approach," Econ. Lett. 64, 279-283.

Kukushkin, N.S. (2000a). "Potentials for Binary Relations and Systems of Reactions," Moscow: Russian Academy of Sciences, Computing Center.

Kukushkin, N.S. (2000b). "Nash Equilibrium in Games with Additive Aggregation," Ekonomika i Matematicheskie Metody 36(4), 58-66 (in Russian).

McManus, M. (1962). "Numbers and Size in Cournot Oligopoly," Yorkshire Bull. Econ. Soc. Res. 14, 14-22.

McManus, M. (1964). "Equilibrium, Number and Size in Cournot Oligopoly," Yorkshire Bull. Econ. Soc. Res. 16, 68-75. 
Milchtaich, I. (1996). "Congestion Games with Player-Specific Payoff Functions," Games Econ. Behav. 13, 111-124.

Milgrom, P., and Roberts, J. (1990). "Rationalizability, Learning, and Equilibrium in Games with Strategic Complementarities," Econometrica 58, 1255-1277.

Milgrom, P., and Shannon, C. (1994). "Monotone Comparative Statics," Econometrica $62,157-180$.

Monderer, D., and Shapley, L.S. (1996a). "Potential Games," Games Econ. Behav. 14, 124-143.

Monderer, D., and Shapley, L.S. (1996b). "Fictitious Play Property for Games with Identical Interests," J. Econ. Theory 68, 258-265.

Novshek, W. (1985). "On the Existence of Cournot Equilibrium," Rev. Econ. Stud. 52, 85-98.

Rand, D. (1978). "Exotic Phenomena in Games and Duopoly Models," J. Math. Econ. 5, 173-184.

Sela, A. (1992). "Learning Processes in Games," M. Sc. Thesis. The Technion, Haifa (in Hebrew).

Selten, R. (1970). Preispolitik der Mehrproduktenunternehmung in der statischen Theorie, Berlin: Springer-Verlag.

Tarski, A. (1955). "A Lattice-Theoretical Fixpoint Theorem and its Applications," Pacific J. Math. 5, 285-309.

Topkis, D.M. (1978). "Minimizing a Submodular Function on a Lattice," Oper. Res. 26, 305-321.

Topkis, D.M. (1979). "Equilibrium Points in Nonzero-Sum n-Person Submodular Games," SIAM J. Control Optim. 17, 773-787.

Vives, X. (1990). "Nash Equilibrium with Strategic Complementarities," J. Math. Econ. 19, 305-321.

Voorneveld, M. (2000). "Best-Response Potential Games," Econ. Lett. 66, 289295. 